\title{
Research Progress of New Technology of Mango Deep Processing
}

\author{
$\mathrm{Li} \mathrm{Li}^{1,2}$, Changbao $\mathrm{Li}^{1,2}$,Jian Sun ${ }^{1,2, *}$, Jinfeng Sheng ${ }^{1,2}$, Xuemei He ${ }^{1}$, Dongning Ling ${ }^{1}$, Fengjin \\ Zheng ${ }^{1}$, Ping Yi ${ }^{1}$,Guoming Liu ${ }^{1}$, Jiemin $\mathrm{Li}^{1,2}$,Ming Xin ${ }^{1}$, Yangyuan Tang ${ }^{1}$ \\ ${ }^{1}$ Agro-food Science and Technology Research Institute, Guangxi Academy of Agricultural Sciences, 174 \\ East Daxue Road, Nanning, China, 530007 \\ ${ }^{2}$ Guangxi Key Laboratory of Fruits and Vegetables Storage-processing Technology, 174 East Daxue Road, \\ Nanning, China, 530007
}

Keywords: Mango; Processing New Technologies; Side-Products; Comprehensive Utilization

\begin{abstract}
Mango is a famous tropical fruit, unique flavor, nutrient-rich. This paper reviews the research results of mango processing and comprehensive utilization in recent years, including mango puree, new health drink, fruit wine, fruit vinegar, preserved fruit and jam, as well as processing products such as mango peel and fruit to provide a theoretical basis for further development for the comprehensive utilization of mango processing products.
\end{abstract}

\section{Introduction}

Mango is a famous tropical fruit, its delicate flesh, unique flavor and nutrient-rich, known as the "tropical fruit king" [1]. At present, China has cultivated and introduced more than 100 varieties of Mango, in Yunnan, Hainan, Guangdong, Guangxi, Fujian, Taiwan and other regions. Harvest period of mango is short, which leads to mechanical damage and rot more easily. This problem not only limits the development of mango market, but also seriously affects the mango production and operation of the enthusiasm and the development of commercial production. At present, mango is mainly sold in China except for a small amount of fruit is used for processing juice, pulp, and preserved fruit. With the mango planting area continues to expand, the output is getting higher and higher, the price is cheaper. Therefore, one of the most effective way is to develop mango and the products processing industry, to solve the problem of mango storage and transportation and promote the rapid development of mango planting industry.

This paper summarizes the latest research progress on the comprehensive utilization of new technology and byproducts in mango processing to further explores new development approaches and improves the sustainable economic and comprehensive utilization of mango industry .

\section{Study on New Technology of Mango Processing}

At present, there are the following problems in the processing of mango: mango has different shapes and the large number of fibers are around the flat core and peel, the general machinery is difficult to obtain a higher yield of mango juice. The pectin content of mango is high, and its processing flow ability is poor. Color of mango changes easily, if proper measures are not adopted, the product will become grey, which will affect the quality of products. Flavor of mango is very easy to loss, and some components are sensitive to heat etc. To solve the above problems, a lot of research has been done on the processing technology of mango to improve the quality and processing advantages of mango and lay a theoretical foundation for the development and utilization of mango as an important food resource.

Mango Puree. Mango harvest period is short, a large number of surplus mature mango fruits are processed into mango puree as soon as possible, preserved for the future processing of mango drinks, mango sauce, mango wine and other mango products to provide the necessary raw materials. The rapid processing of mango puree is one of the most effective ways to save mango, and it is one 
of the most popular methods of fruit preservation in the world. Traditional mango pulp production process: mature mango - cleaning - fruit selection - beating - color - grinding - filtration deployment - degassing - sterilization - packaging - cooling - finished storage. Beating and deployment is the key technology in production, but the rate of pulp is low by traditional beating method. Some domestic mango pulp production plants use the improved method of double-channel beater, but the pulp rate is still only $50-55 \%$. Sreenath et al. studied the effects of various cellulases and pectinases on the viscosity of mango pulp[2]. It was found that ultrazym 100 produced in denmark had the best effect on reducing the viscosity of mango pulp and reduced the viscosity by $82 \%$. Githa et al. found that enzymatic treatment increased the yield of mango pulp by more than $10 \%$ due to enzymatic treatment of decomposing soluble pectin and destroying the cell wall to release cell contents[3]. In recent years, the domestic enzymatic hydrolysis technology has also been studied in the mango juice production process by adding pectinase and cellulase composition of the compound enzyme, which can improve the mango beating pulp rate of $9.0 \%$ or more, enzymatic treatment increased pulp rate, compared to the methods of general single-channel, double-channel beating. And the method can reduce the viscosity, and is easy to operate.The application of enzymatic treatment can be achieved significant economic benefits In the actual production.

The traditional method of sterilization of pulp is the use of thermal sterilization. Although it can extend the shelf life of the product, in varying degrees it destroyed the heat-sensitive nutrients in mango, changed the color of the pulp, led to bad taste and odor, and affected the quality of the product. The results show that the ultra-high pressure treatment sample retains the original color, the reduction rate of the reduced Vitamin C (VC) is $91.18 \%$.Ultra high pressure technology (UHPT) not only has good bactericidal effect, but also ensures the quality of mango puree to the maximum extent.

New Health Drink. Drink is the main processing products in mango and there are mainly mango milk drinks, compound drinks, pulp drinks and so on. With the development of the global economy and social progress, people put forward the higher requirements for the quality of food.s Pure natural, eutrophic, less pigment and no additives become a new requirement for modern drinks. Nutrient-rich, with the body needs a variety of vitamins, trace elements, amino acids, minerals, is the ideal natural drinks, so that the development of functional fruit juice drinks become a new market-oriented beverage industry. Wu Xianhui et al. developed a glucomannan functional milk drink with health care function and good taste [4]. Through the study of processing technology of mango and aloe compound health drink, Yang Zhengxing et al. developed a unique mango and aloe complex health drink[5].

Fruit Wine. Fruit wine is a low degree of wine with a good flavor and a high nutritional value. In the process of mango picking, storage and processing, damaged mango can not be processed into a complete product, but be made of fruit wine to improve the utilization of raw materials and enrich the types of mango processed products. Wang Chunyan et al. studied the fermentation process of mango wine. The best fermentation temperature was $20^{\circ} \mathrm{C}$, the optimum dosage of sodium sulfite was $40 \mathrm{mg} / \mathrm{L}$, and the optimum sugar content was $19 \%$, making its products clear, transparent, low alcohol, with color and flavor of the original fruit and preservation of the natural nutrition. The results showed that $\mathrm{SO}_{2}(100 \mathrm{mg} / \mathrm{L})$ was sterilized, the sugar content was $25 \%$, and the content of sweetened mango wine was 3 times higher than that of the control. $\mathrm{pH} 4.5$, inoculation amount of $5 \%$, in Chinese southern summer temperature conditions, the fermentation can be made of natural fruit, fruit color and unique flavor of sweet mango wine. The optimal fermentation conditions of mango wine were $2 \%$ of yeast, $12 \%$ of initial sugar content, $32{ }^{\circ} \mathrm{C}$ and $\mathrm{pH} 4.0$, and the fermented fruit was mixed with fruit liquor. And aging, and ultimately get the flavor of flavor and flavor of mango wine. Li Shanxiong for the quality of mango wine research, brewed with mango natural flavor, full bodied, nutrient-rich fermented mango wine. Lu Xiyu developed the fermentation process of mango and papaya compound fermented fruit wine, using enzymatic treatment. After fermented and aged, the mango and papaya compound wine was $12.1 \%$. Reddy et al. used pectinase to treat mango wine after juice treatment, and studied the characteristics and aroma components of 
mango wine. The results showed that the content of aromatic compounds in mango wine was similar to that of wine. Further studies have showed that pectinase treatment could improve the yield of fruit juice and increase the alcohol content of mango wine [6].

At present, a lot of studies have been done on the ingredients of mango wine at home and abroad, and the volatile compounds of mango wine have been analyzed. According to the qualitative and quantitative analysis of 102 kinds of volatile compounds, it was found that isoamyl alcohol and 2-phenylethanol were the main components, and the the compounds which contributed most to the aroma of mango wine were ethyl butyric and decyl aldehyde.Li et al. compared the effects of three different Saccharomyces cerevisiae on the chemical composition and aroma components of the wine, indicating that fruit wine made from different Saccharomyces cerevisiae had different characteristics.

Fruit Vinegar. Mango vinegar is a liquid sour taste seasoning made from mango by microbial fermentation. Fermented mango vinegar is sweet, sour taste, rich in flavor and containing a variety of amino acids, vitamins and minerals needed by the human body. It has the functions of improving the body immunity, reducing blood lipids, lowering blood pressure and delaying senility. Qin Yan et al. have studied the mango fruit vinegar production process, showing that the semi-solid fruit juice was good for alcohol fermentation, making the fermentation powerand yeast adaptability. The best conditions for acetic acid fermentation is at the temperature of $35^{\circ} \mathrm{C}$, the initial $\mathrm{pH} 3.7,7 \%$ of the initial alcohol and 10\% of inoculation amount of acetic acid bacteria. Zhang Bin et al. made mango fruit vinegar by alcoholic fermentation and acetic acid fermentation, and added $0.15-0.2 \%$ pectinase for enzymatic treatment to solve the problem that mango contained a large number of pectin and was not conducive to fermentation. The ethanol fermentation by immobilized yeast can shorten the time of clarification, and make the product pure and honey sweet. The mass fraction of mango fruit vinegar acetic acid was $5-7 \%$, the content of $\mathrm{VC}$ was $\geq 30 \mathrm{mg} / \mathrm{L}$ and the content of soluble solid was $3.0 \%$. At the same time, it was found that the concentration of total acid could be increased by ventilating and stirring. Aeration, mixing and splitting, adding mash can shorten the fermentation time and increase the conversion rate. Sun Feifei, et al. developed honey taste, trehalose taste, isomaltulose taste mango vinegar nutritious drinks, with sweet and sour palatability, soft taste, unique flavor and rich nutrition [7].

Preserved Fruit. Traditional mango fruit preserves are mostly cooked by boiling. The products are dark in color, soft in flesh and high-sugar foods with sugar content of more than $65 \%$ so that they do not meet the requirements of modern healthy diet. Wang Tianlu studied the processing technology of low-sugar mango preserved fruit. The mango slices hardened and colored for $6 \mathrm{~h}$ with compound $0.2 \%$ citric acid, $0.3 \%$ calcium chloride and $0.2 \%$ sodium sulfite, and then sugared for 8 hd at sugar concentration of $40 \%$ and then carry out vacuum drying after $3 \mathrm{~h}$ of vacuum sugar infiltration. Wang Zhongfeng improved the production process of low-sugar mango fruit, with $0.5 \% \mathrm{CaCl}_{2}, 1.0 \% \mathrm{NaCl}, 0.2 \%$ citric acid combined solution to protect the mango slices, adding $0.5 \%$ konjac flour to increase the fullness of the product, at $0.06 \mathrm{MPa}$ for $30 \mathrm{~min}$, room temperature sugar infiltration for $4 \mathrm{~h}, 3$ times repeating. The final product contained only $35-40 \%$ of sugar content, which meet the requirements of low-sugar preserved fruit. Feng Chunmei et al. studied the processing technology of raw salt-free mango dry, and soaked the mango slices with 1: 3 mass ratio of sugar and mango. The initial temperature of soaking sugar was $30^{\circ} \mathrm{C}$, the initial concentration of sugar was $30^{\circ}$ Brix, D-sodium ascorbate dosage of $0.3 \%$ and maintain a vacuum at $0.07 \mathrm{MPa} 3 \mathrm{~h}$, with $60-70{ }^{\circ} \mathrm{C}$ hot air drying to non-stick so far, that is finished mango dry. Sheng Jinfeng et al. carried on the experiments on the permeability of mango under the conditions of atmospheric pressure, ultrasonic, microwave and vacuum, the results showed that the microwave method was most conducive to the permeability of mango fruit preserved [8].

Jam. Low-sugar mango jam is a kind of young and old food. Its unique flavor, good appearance and good preservationmakes it strong market competitiveness. Low-sugar mango jam can be used not only for families or restaurants to eat directly, but also as a filling for making cakes or pastries.. The processing technology for mango low-sugar jam is adding thickener $0.2 \%$, the ratio of jam and sugar 5: 2 , and citric acid $0.15 \%$. The resulting jam is good in body, delicate in texture, 
golden yellow, and rich in mango flavor, sweet and sour, nutritious.

Expanded Food. Fruit and vegetable crisp is a new form of fruit and vegetable processing developed in recent years. At present, the processing of fruit and vegetable crisp film is mainly used in the vacuum frying method. The frying costs a lot of edible oil, and the crisp contains high oil content of more than $20 \%$. Long-term consumption can cause problems for people's health, and frying will cause oil rancidity and short shelf life. The optimum conditions for the microwave puffing of mango slices were as follows: using pre-drying, the moisture content of the mango fruit before puffing was reduced to $12 \%$, and then the products were obtained by microwave puffing $22 \mathrm{~s}$. The swelling rate, crispness, color and appearance of the mango slices were all good. The technology of explosion puffing drying at modified temperature and pressure is a new method to develop mango crisp film. The produced fruit and vegetable chips have the advantages of green natural, nutrient rich, crisp and delicious, convenient and easy to store.

\section{Mango Comprehensive Utilization}

Mango Kernel. Researches have shown that antibacterial ingredients can be isolated from mango kernel, with high antioxidant activity, free radical scavenging and other biological functions. Liu Xin et al. studied the extraction of procyanidins from mango kernel by ultrasonic cell pulverizer and obtained the best extraction conditions. The extraction rate of procyanidins was $91.4 \%$ of that in mango kernel. Gao Yuntao et al. proposed a new method for the extraction of polyphenols from mango kernel by ultrasonic extraction with propanol-ammonium sulfate aqueous two-phase system (ATPS). The results showed that the polyphenol content of the extract obtained by ultrasonic assisted ATPS was higher than that of conventional ultrasonic extraction. Studies have showed that mango nuclear extract had obvious bacteriostatic effect in vivo and in vitro on common intestinal infection pathogens. The results showed that the ethanol extract of mango cultivars had certain scavenging DPPH free radicals, abolished $\mathrm{ABTS}^{+} \cdot \bullet \mathrm{Fe}^{3+}$ reducing force, anti-lipid oxidation and $\mathrm{Fe}^{2+}$ network, according to the studies on the antioxidant activity of the three varieties of mango fruit extract. Narasimha et al. have found that the fat can be refined from mango kernel, with potential edible value, and used as a substitute for butter or cocoa butter.

Mango Peel. Mango peel as by-products in mango industrial processing production, are generally abandoned, not only causing great waste of resources, but also polluting the environment. In recent years, domestic and foreign scholars have made a lot of researches on the active ingredients of mango peel, and established a new technology to extract effective functional factors from mango by-products. Mango peel contains flavonoids, polyphenols, organic acids and a variety of volatile oil and other health food active ingredients, and they have the physiological functions of scavenging oxygen free radicals, enhancing immune regulation, relieving cough and resolving phlegm and anti-inflammatory effects. After separation, they can be applied to the formulation design of health foods or the development of new health food, such as delaying aging, regulating blood lipids, enhancing physical fitness. Huang Minqi et al. extracted mangiferin from the mango peel for the first time, with a yield of $2.3 \%$, and use a variety of models to show that it has antitussive, expectorant and anti-inflammatory effects. Song et al. studied the extraction of aroma compounds from mango peel by ultrasonic assisted extraction method and prepared mango microcapsules with gelatin and sodium alginate. Berardini et al. extracted pectin and polyphenol compounds from mango peel, and found that the antioxidant activity of extracts was overmangiferin and quercetin 3-O-glycosides [9].

Study on the Processing of Mango Fruit Drop and Thinning Fruit. In recent years, China has taken some fine management measures on mango, such as: flowers, thinning, mango bagging technology, so that the amount of fruit per plant can be effectively controlled to enhance the quality of fruit. The total amount of drop and thinning fruits is about $70 \%$ of the output of mango. Except for few used as feed for farmers, most of these resources were discarded as wastes. Especially mangoes that fall in orchards, they also induced insect pests like bactrocera dorsalis and mango fruit weevil, which harmed mango growth, affected mango quality. Therefore, the development and utilization of mango fruit drop, thinning fruit and green mango can not only make waste profitable, 
increase the income of farmers, but also improve the orchard sanitation and the proportion of high quality mango.

The processing of low-sugar preserved fruit can provide a way for the utilization of mango fruit drop. Zhang Yan et al. explored the processing technology of low-sugar preserved fruit with mango fruit drop, using three times vacuum sugar infiltration, by adding $1.0 \%$ citric acid and $0.5 \%$ gelatin in the sugar solution, and obtained excellent quality and flavor of low-sugar mango preserved fruit.. Guo An et al. discussed the processing technology of the mango pickles, the hot and sour mango piece and the spiced mango, using mango fruit drop and thinning fruit as raw materials. Make appetizing food has the characteristics of cheap raw materials, good texture, flavor and simple process, easy to popularize in mango producing areas [10].

\section{Conclusion}

Mango is a famous tropical fruit, with the characteristics of wide adaptability, fast growing, early fruit, high yield, long fruiting stage and high economic value. China is abundant in mango resources. The comprehensive utilization of mango and the development of new products not only meet the development trend of modern food, but also give full play to the advantages of resources in Chinese fruit and vegetable industry. However, the deep processing of mango and comprehensive utilization are still in its infancy. Few varieties and unstable quality of products also affect the further development of mango industry. Therefore, it is of great significance to explore functional active ingredients and their mechanisms of action in mango and by-products, and to study the practical and comprehensive utilization methods for developing the industrial chain of mango and promoting the development of economy.

\section{Acknowledgements}

Fund Project: This research was supported by Special Fund for Agro-scientific Research in the Public Interest (Grant No. 201303073) ,National Natural Science Foundation of China (Grant Nos. 31160407, 31000927, 31560467, 31660589, 31560006), Bagui Scholars Project Special Fund (Grant No. [2016]21), Technology Foundation for Selected Overseas Chinese Scholar, Ministry of Personnel of China (Grant No. Ren She Ting Han [2015]192), Guangxi Natural Science Foundation (Grant No. 2014GXNSFDA118013, 2015GXNSFBA139102), Guangxi Agricultural Key Science and Technology Program (Grant No. 201527), Guangxi Scientific Research and Technological Development Projects (Grant No. Gui Ke AD16380015) and Foundation of Fundamental Research Project from Guangxi Academy of Agricultural Sciences (Grant Nos. 2016018, 2015YT86, 2015JM14, 2015JZ75, 2014YQ05).

\section{References}

[1] Masibo, M. \& He, Q. Major mango polyphenols and their potential significance to human health[J]. Comprehensive Reviews in Food Science and Food Safety, 2008, 7:309-319

[2] Sreenath H K,Nanjundaswamy A M,Sreekantiah K R.Effect of various cellulases and Pectinases on viscosity reduction of mango pulp[J].Journal of Food Science, 1987,52(1):230-231

[3] Githaiti J K.Application of pectase in the preduction of mango fruit[J]..Acta Alimentaria, 1991, 20(2):97-102.

[4] Wu Xianhui, Yin Na, Luo Yu-nong, Yao Min-na. Study on Functional Glucomannan Mango Juice Milk Beverage [J]. Journal of Southwest University (Natural Science Edition), 2013, 35 (8): 150-154.

[5] Yang Zhengxing, Yin Xiuhua, Feng Yan. Mango aloe vera compound health drink [J]. Guangxi Horticulture, 2003, 46 (1): 6-9. 
[6] Reddy, L. V. A. Reddy, O. V. S. Effect of enzyme maceration on synthesis of higher alcohols during mango wine fermentation[J]. Journal Food Quality, 2009, 32:34-47.

[7] Sun Feifei, Qin Yan, Wei Xingming. Three kinds of taste mango vinegar nutrition beverage formula design [J]. Guangxi Science, 2008, 15 (3): 321-324.

[8] Sheng Jinfeng, Li Li, Sun Jian, Li Changbao, Li Jiemin, Zheng Fengjin, Liu Guoming, He Xuemei, Liao Fen, Zhang Yayuan, Effects of different osmotic methods on the quality and tissue cells of mango fruits, Modern Food Science and Technology[J]. 2014, 6 (30).202-206

[9] Berardini N., Matthias K., Andreas S.Utilization of mango as a source of pectin and polyphenolics [J] . Innovative Food Science and Emerging Technolog ies, 2005, 6: 442- 452

[10] Guo An, Zhao Yi. Mango fruit drop, fruit processing of food processing technology [J]. Food Research and Development, 2007, 28 (10): 128-130. 